Published in final edited form as:

Nature. 2020 July ; 583(7817): 542-547. doi:10.1038/s41586-020-2495-2.

\title{
Cleavable Comonomers Enable Degradable, Recyclable Thermoset Plastics
}

\author{
Peyton Shieh $^{1}$, Wenxu Zhang ${ }^{1}$, Keith E. L. Husted ${ }^{1}$, Samantha L. Kristufek ${ }^{1}$, Boya Xiong ${ }^{2}$, \\ David J. Lundberg ${ }^{1,3}$, Jet Lem ${ }^{1,4}$, David Veysset ${ }^{1}$, Yuchen Sun ${ }^{1,4}$, Keith A. Nelson ${ }^{1,4}$, \\ Desiree L. Plata ${ }^{2}$, Jeremiah A. Johnson ${ }^{1}$ \\ ${ }^{1}$ Department of Chemistry, Massachusetts Institute of Technology, 77 Massachusetts Avenue, \\ Cambridge, MA 02139, United States \\ 2Department of Civil and Environmental Engineering, Massachusetts Institute of Technology, 77 \\ Massachusetts Avenue, Cambridge, MA 02139, United States \\ ${ }^{3}$ Department of Chemical Engineering, Massachusetts Institute of Technology, 77 Massachusetts \\ Avenue, Cambridge, MA 02139, United States \\ ${ }^{4}$ Institute for Soldier Nanotechnologies, Massachusetts Institute of Technology, 77 Massachusetts \\ Avenue, Cambridge, MA 02139, United States
}

\section{Summary}

Thermosets play a key role in the modern plastics and rubber industries, comprising $\sim 20 \%$ of polymeric materials with a worldwide annual production of $\sim 65$ million tons. ${ }^{1,2}$ The high density of crosslinks that gives thermosets their useful properties (e.g., chemical/thermal resistance, and tensile strength) comes at the expense of degradability and recyclability. Here, using the industrial thermoset polydicyclopentadiene (pDCPD) as a model system, we show that when a small number of cleavable bonds are selectively installed within the strands of thermoset plastics, the resulting materials can display the same mechanical properties as the native material, yet they are able to undergo triggered degradation to yield soluble, recyclable products of controlled size and functionality. In contrast, installation of cleavable crosslinks, even at comparably high loadings, does not produce degradable materials. These findings reveal cleavable bond location as a design principle for controlled thermoset degradation. Moreover, a new class of recyclable thermosets poised for rapid deployment is introduced.

Existing strategies to create reprocessable thermosets have focused on dynamic covalent bond exchange, ${ }^{3-8}$ thermomechanical degradation, ${ }^{9}$ or wholesale replacement of network components with degradable versions. ${ }^{10-13}$ We hypothesized that optimizing the location of

\footnotetext{
Users may view, print, copy, and download text and data-mine the content in such documents, for the purposes of academic research, subject always to the full Conditions of use:http://www.nature.com/authors/editorial_policies/license.html\#terms

Correspondence and requests for materials should be addressed to J.A.J (jaj2109@ mit.edu).

Author contributions

P.S. and J.A.J. conceived of the idea. P.S., W.Z., and K.H. synthesized materials. P.S., W.Z., S.L.K., and K.E.L.H. characterized materials. D.J.L., P.S., and J.A.J. developed the theoretical framework. B.X. and D.L.P. conducted accelerated weathering experiments. D.J.L. performed techno-economic analyses. J.L., D.V., Y.S. and K.A.N. designed and conducted microparticle impact experiments. P.S. and J.A.J. wrote the manuscript. All authors read and revised the manuscript.
} 
cleavable bonds within thermosets may provide a complementary approach, enabling the mild, chemically-triggered degradation and recycling of thermosets at low cleavable bond loadings. Here, leveraging $\mathrm{pDCPD}^{14-16}$ and recent advances in ring-opening metathesis polymerization (ROMP) comonomer design, ${ }^{17}$ we establish that introducing cleavable bonds within the strands of covalently crosslinked thermoset plastics can impart degradability and potential recyclability at low co-monomer loadings while the analogous addition of cleavable crosslinks cannot. This design principle may apply to other materials formed via crosslinking of reactive polymers (Fig. 1a) including acrylics, styrene-divinylbenzene, vulcanized rubber, silicones, etc., suggesting that it could motivate searches for novel cleavable comonomers and potentially contribute to the design of sustainable plastics and elastomers. ${ }^{18-22}$

\section{Theoretical Framework}

To estimate the amount of cleavable comonomer $x$ required to degrade networks of strands with $f$ crosslinkable functional groups and $c$ crosslinks into soluble products, we derived a reverse gel-point model based on Miller-Macosko ${ }^{23}$ and Flory-Stockmayer ${ }^{24,25}$ theories (Fig. 1b, Extended Data Fig. 1; see Methods):

$$
x>\frac{c(2 f-1)-f}{c+f}
$$

From this equation, it is observed that when $f \gg>c$, degradation into soluble products is expected when $x>\sim 2 c$. If we instead assume that $f \approx c$, then degradation to soluble products is expected when:

$$
x>c-1
$$

Thus, the model predicts what is also intuitive: degradation of thermosets to soluble products can be achieved when the number of cleavable bonds in strands is similar in magnitude to the number of crosslinks. We note that this model assumes that the cleavable bonds are randomly distributed along strands, that equivalent functional groups have equal and independent reactivity, and that there are no intramolecular reactions; thus, it only provides an estimate of $x$-the presence of loops that consume functionality yet do not contribute to gelation will further lower the number of degradable bonds needed to achieve degradation (vide infra). ${ }^{26}$

\section{Cleavable Bond Location Controls Degradability}

To test the validity of this model, we leveraged recently reported silyl ether monomers that copolymerize with norbornene derivatives by ROMP to produce degradable statistical copolymers. ${ }^{17}$ Here, DCPD was mixed with different amounts of the silyl ether monomer iPrSi $(0,5,10$, or $15 \% \mathrm{v} / \mathrm{v}$ or one equivalent of iPrSi per 30.8, 14.6, and 9.2 equivalents of DCPD, respectively, Fig. 1c); the mixtures were cured in the presence of Grubbs $2^{\text {nd }}$ generation ROMP initiator. While this curing protocol was not further optimized, the resulting materials displayed Young's moduli in the GPa range (vide infra) as expected for 
pDCPD thermosets. In the initial stage of pDCPD curing, the norbornene component of DCPD copolymerizes with iPrSi to form linear polymer strands with $f$ cyclopentene sidechains as potential crosslinking sites and $x$ cleavable silyl ether linkages (Fig. 1c). Cyclopentene sidechain metathesis leads to iPrSi-doped pDCPD with $c$ crosslinks. Silyl ether cleavage is expected to generate fragments with $\langle c \mid x+1\rangle$ crosslinks per strand; thus, as $x$ approaches $c$, smaller soluble products should be produced. We note that this approach stands in contrast to the indiscriminate degradation of thermosets, which produces products of uncontrolled size and chemical composition that typically have much lower value; the comonomer strategy provides a way to trigger thermoset degradation at low comonomer loadings and control degradation product size and composition (vide infra). Lastly, we note that a preliminary techno-economic analysis suggested that iPrSi can be manufactured inexpensively, making it potentially feasible for large-scale use especially if added as an additive to existing pDCPD workflows (Fig. S1, Table S1).

To compare iPrSi-doped pDCPD to analogs with cleavable crosslinks, pDCPD samples were prepared in the presence of up to $80 \% \mathrm{v} / \mathrm{v}$ of a bis-cyclooctene crosslinker featuring a silyl ether linkage (SiXL, Fig. 1d, S2-S3). In this case, the addition of $y$ cleavable crosslinks (from SiXL) is expected to yield thermosets with $c+y$ crosslinks; cleavage of the $y$ linkages leaves a network with $c$ crosslinks, precluding the formation of soluble products for all $y$ values other than those much greater than $c$ (Fig. 1d). We note that in thermosetting materials with mostly cleavable crosslinks, theoretically it is possible, though often difficult in practice, to achieve material degradation once nearly all of the crosslinks are cleaved. ${ }^{9}$ For thermosets that lack naturally cleavable crosslinks, however, the addition of a small number of cleavable crosslinks is not expected to produce degradable materials.

To test this hypothesis, samples of iPrSi-doped, SiXL-doped, and native pDCPD (Fig. 2a, Extended Data Fig. 2) were exposed to an excess of tetrabutylammonium fluoride (TBAF), a fluoride reagent that selectively cleaves silyl ethers and is often used to etch silicone elastomers, ${ }^{27}$ at room temperature. After $12 \mathrm{~h}$, the native pDCPD remained fully intact (Fig. $2 b$ ). In contrast, samples with only 10 or $15 \%$ v/v iPrSi dissolved (Fig. 2a, 2b). While the $5 \%$ iPrSi-doped sample remained intact, it was noticeably swollen, suggesting a decrease in crosslink density that was further confirmed by analysis of Si content using ICP-OES (Fig. S4). In each case, degradation of the iPrSi-doped samples occurred over the course of $\sim 4 \mathrm{~h}$, presumably limited by fluoride diffusion into the materials (Fig. S5). Meanwhile, SiXLdoped materials did not degrade into soluble products even at $80 \%$ SiXL loading (Fig. 2b, Extended Data Fig. 2, Figs. S6-S7). The rate of SiXL cleavage by fluoride was observed to be similar to that of iPrSi (Fig. S8), suggesting that this difference in extent of degradation is due to network topology.

To further compare the roles of cleavable strands versus crosslinks in these materials, samples of 5\% v/v and 10\% v/v iPrSi and 20\% v/v SiXL doped pDCPD were swollen to equilibrium in tetrahydrofuran (THF) and characterized by oscillatory rheology (Figs. 2c, Extended Data Fig. 3, Fig. S9). Strikingly, treatment of the iPrSi-doped samples with TBAF led to large decreases in storage modulus ( 100-fold for 5\% iPrSi doped pDCPD) while the modulus of the $20 \% \mathrm{v} / \mathrm{v}$ SiXL-doped sample decreased by only $\sim 5$-fold. 


\section{Functional Evaluation of Degradable pDCPD}

Next, we studied the functional performance of iPrSi-doped pDCPD for comparison to the native material. In tensile tests, the $10 \%$ and $20 \%$ iPrSi-doped samples showed nearly identical Young's moduli and elongations at break compared to pDCPD (Figs. 3a-c, S10S11) while 33\% and 50\% iPrSi-doped samples showed much lower moduli. These results were corroborated by dynamic mechanical analyses (DMA) and nanoindentation studies (Figs. 3d-e, S12-S13). Thermal gravimetric analysis (TGA) showed similar decomposition temperatures for all samples (Fig. 3f). DMA showed a modest decline in $\mathrm{T}_{\mathrm{g}}$ from $166^{\circ} \mathrm{C}$ for native pDCPD to $138{ }^{\circ} \mathrm{C}$ for the $10 \% \mathrm{iPrSi}$-doped sample and a further $\mathrm{T}_{\mathrm{g}}$ lowering for higher iPrSi loadings (Fig. S12, Extended Data Fig. 4a). We note that $33 \%$ and $50 \%$ iPrSidoped samples exhibit $\mathrm{T}_{\mathrm{g}}$ values closer to or below room temperature $\left(46^{\circ} \mathrm{C}\right.$ and $14{ }^{\circ} \mathrm{C}$, respectively), which accounts for their lower moduli as measured by tensile testing at room temperature. In support of this notion, all of the samples displayed similar moduli at $\mathrm{T}_{\mathrm{g}}-60$ ${ }^{\circ} \mathrm{C}$ as measured by DMA (Extended Data Fig. 4b).

Outstanding ballistic impact resistance is one of the most well-known properties of pDCPD.

${ }^{28}$ To assess the ballistic impact response of our degradable analogues, laser induced projectile impact tests (LIPIT) were conducted on $10 \%$ iPrSi-doped and native pDCPD films $(23.0 \pm 1.7 \mu \mathrm{m})$ using steel microparticles $(12.8 \pm 0.4 \mu \mathrm{m}$ diameter $) .{ }^{29}$ High-speed imaging revealed that films of $10 \%$ iPrSi-doped material stopped projectiles with the same efficiency as native pDCPD (Fig. 3g, Figs. S14-S17, Table S2, S3). The coefficient of restitution $(\mathrm{CoR})$, defined as the ratio of rebound velocity to impact velocity, was similar for these materials across impact velocity regimes of particle rebound, embedment, and film perforation (Fig. 3h), indicating indistinguishable high strain-rate responses.

We next sought to probe the degradation of these materials in more detail. In addition to TBAF, which is convenient for laboratory scale reactions, hydrofluoric acid, which is used to etch silicon on large scale in the semiconductor industry, readily dissolved our iPrSi doped pDCPD at room temperature (Fig. S18). Silyl ethers are also susceptible to cleavage under acidic or basic conditions ${ }^{30}$; however, due to their hydrophobic nature, iPrSi-doped pDCPD samples displayed sluggish hydrolysis in aqueous acidic $(\mathrm{pH}=0)$ or basic $(\mathrm{pH}=14)$ conditions, though they did show evidence of surface etching following exposure to aqueous sodium hydroxide for 30 minutes (Fig. S19, S20). To demonstrate tuning the degradation of these materials, we prepared samples doped with $10 \%$ v/v of EtSi, a comonomer significantly more susceptible to hydrolysis. ${ }^{17}$ These materials displayed enhanced degradation under mixed aqueous/organic acidic conditions (Extended Data Fig. 5).We envision that through the use of a wider range of comonomers, it may be possible to generate thermosets with variable degradation rates and mechanisms (e.g., photochemical, enzymatic, etc.). ${ }^{31}$

Finally, given concerns over the accidental release of plastic waste into the natural environment, ${ }^{32}$ we studied degradation of $\mathbf{~ P r S i - ~ a n d ~ E t S i - d o p e d ~ p D C P D ~ e x p o s e d ~ t o ~}$ synthetic seawater and ultraviolet light for $16 \mathrm{~d}$ (Figs 3i, Extended Data Fig. 6, Tables S4, S5). Significant increases (up to $\sim 2-3$ fold) in the extent of degradation relative to native pDCPD were observed. Transmission electron microscopy (TEM) revealed the presence of 
sub-5-nm particles following degradation (Fig. S21). While the generation of microplastics (typically $\mu \mathrm{m}$-mm-range particles) may be a concern, ${ }^{33}$ nanoscale plastics could be important intermediates that enhance the total degradation rate of bulk plastics. Notably, optimization of the size and composition of pDCPD degradation products can be achieved by tuning the silyl ether monomer substituents and loading, which is challenging for less selective degradation processes.

\section{Characterization and Reprocessing of Products}

The degradation products of iPrSi-doped pDCPD are hydroxylated polymers bearing cyclopentene functionalities that could be used for recycling or repurposing (Fig. 4a). To demonstrate this concept, samples of 10, 20,33, or 50\% iPrSi-doped pDCPD were prepared and subjected to degradation using TBAF (Fig. S22). The resulting soluble products were characterized by 1-D and 2-D solution-state NMR, including ${ }^{1} \mathrm{H},{ }^{13} \mathrm{C}, \mathrm{COSY}, \mathrm{HSQC}$, HMBC, and NOESY (S23-S29) with greatly improved resolution compared to solid-state NMR (Fig. 4b). To enable comparison of the NMR spectra, a sample of linear, noncrosslinked pDCPD was independently prepared (Figs. S30-S31). ${ }^{34}$ From the combination of these studies, a 3:2 ratio of aliphatic to olefin carbons could be assigned in the ${ }^{13} \mathrm{C}$ NMR spectrum of the iPrSi-doped pDCPD degradation products, indicating that $\sim 15 \%$ of the cyclopentenes of the polynorbornene strands had reacted (Fig. 4b). Based on our model (Fig. 1b), a material with $15 \%$ effective crosslinks would require $>15 \%$ cleavable bonds to degrade into soluble products; thus, we estimate that large fraction of the reacted cyclopentene groups in pDCPD are consumed through intramolecular reactions (loops). This insight into the structure of $\mathrm{pDCPD}$, uniquely enabled by the cleavable comonomer approach, lends clear and quantitative support to the model of pDCPD as being crosslinked by secondary metathesis reactions of cyclopentene substituents. ${ }^{35}$

To examine the role of iPrSi loading on degradation product size, the soluble samples prepared above were analyzed by gel permeation chromatography (GPC) (Fig. 4c) and diffusion ordered spectroscopy (DOSY) (Figs. S32-S35), both of which showed an inverse relationship between iPrSi loading and degradation product size. From GPC, the weightaverage molar masses of these samples ranged from 2-8 kDa (Table S6), while DOSY allowed us to estimate that the average diameter of the degradation products of the $10 \%$ iPrSi-doped material was $\sim 4 \mathrm{~nm}$, which is on the length scale of individual polymer strands. This result was further corroborated by TEM imaging (Fig. 4d, Fig. S36).

Given that these degradation products possess many unreacted cyclopentene substituents (Fig. 4a,b), we reasoned that they could be reprocessed into new pDCPD materials. Indeed, mixing the degradation products of our $10 \%$ iPrSi-doped material (25 wt. \%) with DCPD and curing following the same procedure used for native $\mathrm{pDCPD}$ produced recycled samples with comparable stress-strain behavior and elastic moduli (Fig. 4e-f, Figs. S37-S48). Moreover, the recycled samples displayed similar ballistic impact resistance to native pDCPD (See Supporting Information). Finally, carbon fiber composites of pDCPD have been explored for high-performance applications, ${ }^{16}$ but the costly embedded carbon fiber typically cannot be recovered from such materials. When carbon fiber fabrics were embedded into 10\% iPrSi-doped pDCPD, they could be quantitatively recovered (Fig. 4i). 
Raman spectra for pristine versus recovered carbon fiber were very similar, suggesting that the mild pDCPD degradation process has no impact on the fiber composition (Fig. 4j). These results hint at potential opportunities for thermoset composite recycling.

\section{Data availability}

All data supporting the findings of this study are available within the Article and its Supplementary Information, and/or from the corresponding author upon reasonable request.

\section{Methods}

\section{A General Theoretical Framework for Degradable Thermosets via Copolymerization}

Here, we describe the theoretical model for network degradation in further detail. We utilize the following variables, which are consistent with the terminology defined in Fig. 1. Moreover, we introduce an additional variable for the dispersity of the degradation fragments.

$f=$ number-average degrees of polymerization of non-degradable, functional (crosslinkable) monomer (e.g., DCPD)

$c=$ number-average crosslinks per strand (i.e., the number of $f$ groups that have reacted to form crosslinks)

$x=$ number-average degrees of polymerization of degradable comonomer (e.g., iPrSi)

$Ð=$ dispersity of linear fragments obtained after strand degradation in reverse gel-point model

To begin, we assume that the network structure formed by the crosslinking of linear copolymer strands followed by cleavage of degradable bonds in those strands (as is done experimentally in our manuscript) is identical to the network formed by first cleaving the linear copolymer strands and then cross-linking the resulting "fragments" as shown in Extended Data Fig. 1. Then, we leverage classical Flory-Stockmayer and Miller-Macosko theories to determine what values of $x$ would inhibit gelation for given values of $f$ and $c$. As is the case for these classical gelation theories, our model assumes that all functional groups of the same type have equal reactivity, that all functional groups react independently of each other, and that there are no intramolecular reactions. Moreover, we assume that degradable comonomers $x$ are randomly distributed along the strand backbone.

We estimate the number-average degrees of polymerization (DP) of the linear fragments generated after degradable monomer cleavage as:

$$
D P=\frac{f}{x+1}
$$

To provide an estimate of $\oslash$ for these fragments, we applied a Monte Carlo analysis where $x$ degradable co-monomers were randomly incorporated into a linear polymer of DP $=f$ and 
calculated the fragment DP after degradation. We repeated this process $10^{6}$ times to arrive, as expected when $f \gg>x>1,{ }^{36}$ a fragment dispersity of $\sim 2$.

Based on Miller-Macosko theory, we define the critical extent of reaction required for gelation during crosslinking a disperse mixture of polymer strands with a single crosslinking functionality as $p_{c}$ :

$$
p_{c}=\frac{1}{f_{w}-1}
$$

where $f_{W}$ is the weight-average crosslink functionality of the fragments, which is defined as:

$$
f_{w}=\frac{Ð f}{x+1}
$$

We also note that for crosslinked networks below the gel point:

$$
\frac{c}{f}<p_{c}
$$

Combining equations (2) and (4), we obtain:

$$
\frac{c}{f}<\frac{1}{\left(\frac{Ð f}{(x+1)}\right)-1}
$$

Solving for $x$ provides the following relationship:

$$
x>\frac{c(Đ f-1)-f}{c+f}
$$

Assuming $D=2$ arrives at the expression provided in the main text and plotted in Fig. 1B for $f=3000$ :

$$
x>\frac{c(2 f-1)-f}{c+f}
$$

While our model can account for any $f$ or $c$ value, in practice, many materials can be approximated by either of two limiting cases: $f>c$ or $f \sim c$. The limiting case of $f \gg>c$ reflects materials where the number of crosslinks is low relative to the number of potential crosslinkable functionalities. Such is the case for vulcanized thermosets. In contrast, the limiting case of $f \sim c$ corresponds to materials where nearly every crosslinkable functionality is involved in a crosslink, as is found, for example, in many epoxy thermosets.

\section{pDCPD Resin Precursor Preparation}

Dicyclopentadiene (DCPD) and iPrSi were mixed in the desired ratio. Next, finely powdered Grubbs $2^{\text {nd }}$ generation ROMP initiator was dissolved into this mixture at a concentration of $2 \mathrm{mg} / \mathrm{mL}$. The finely powdered initiator was generated by dissolving the commercially obtained Grubbs $2^{\text {nd }}$ generation complex in dichloromethane in a glass vial, evaporation of the solvent under vacuum, and scraping the residue from the side of the vial 
with a spatula. This process enables the rapid and full dissolution of the catalyst in DCPD/ iPrSi mixtures. The solutions remained liquid at room temperature at silyl ether concentrations of $10 \%$ or higher, while solidification occurred at $5 \%$ or lower concentrations. In these cases, the solidified monomer mixture was melted by gentle heating in a water bath $\left(\sim 40^{\circ} \mathrm{C}\right)$. The homogenous pink solutions were used within $5 \mathrm{~min}$ to prepare resins of the desired geometry.

\section{pDCPD Resin Synthesis (Pellets)}

$200 \mu \mathrm{L}$ of the solutions described above were added to $2 \mathrm{~mL}$ flat-bottom screw thread glass vials (VWR, Part No. $46610-772,12 \times 32 \mathrm{~mm}$ ). The vials were heated to $120^{\circ} \mathrm{C}$ for $15 \mathrm{~min}$ in an oven, during which time the pink solution turned into a yellow solid as it polymerized and crosslinked to form $\mathrm{pDCPD}$. The vials were then removed from the oven, cooled to room temperature, and broken with a hammer to release the sample. The collected pDCPD samples were cured for another 30 minutes at $120{ }^{\circ} \mathrm{C}$ and then stored at room temperature until further use.

\section{Laser-Induced Projectile Impact Testing of iPrSi-Doped pDCPD}

Laser induced projectile impact testing (LIPIT) serves as a platform for studying the high strain-rate impact response of materials ${ }^{29}$. LIPIT has been utilized previously to study the impact responses of gels, metals, ceramics, and a range of other materials ${ }^{29,37-40}$. In brief, a high-energy laser pulse (Nd:YAG, $532 \mathrm{~nm}, 10 \mathrm{~ns})$ is focused onto a glass substrate $(210 \mu \mathrm{m})$ coated with an ablative gold layer $(60 \mathrm{~nm})$, and a polyurea film $(40 \mu \mathrm{m})$ - this glass-goldpolyurea configuration will hereafter be referred to as the "launch pad". The launch pad is coated with microparticles and after ablation of the gold layer by a high-energy laser pulse, a particle is propelled at high speeds ranging from tens of $\mathrm{m} / \mathrm{s}$ up to $2 \mathrm{~km} / \mathrm{s}$, with the characteristic strain-rate defined as the impact velocity divided by particle diameter. The projectile speed is varied by adjusting the laser pulse energy. Particle trajectory and impact are captured via an ultra-high-speed camera (SIMX16, Specialized Imaging) with 16 independently triggered CCDs, illuminated by a second pulsed laser ( $640 \mathrm{~nm}, 30 \mu \mathrm{s})$. This provides 16 frames with a minimum exposure time of $5 \mathrm{~ns}$ and varied interframe time. The particle pre-impact velocity $\left(V_{i}\right)$ and post-impact velocity $\left(V_{I}\right)$ are extracted from the image sequences. All particle diameters are measured prior to impact, and film thicknesses are measured with confocal microscopy after impact.

In this experiment, steel microparticles $(12.8 \pm .4 \mu \mathrm{m}$ diameter $)$ were launched with speeds ranging between $150 \pm 3$ and $950 \pm 19 \mathrm{~m} / \mathrm{s}$ at film samples with thicknesses of $23.0 \pm 1.7$ $\mu \mathrm{m}$. Three regimes of impact response were observed: particle rebound, particle embedment, and film perforation. The coefficient of restitution $(\mathrm{CoR})$, the ratio of pre- and post-impact velocities $\left(-V_{I} / V_{i}\right)$, was calculated and plotted to compare the impact responses of the two films. Positive, zero, and negative CoR correspond to particle rebound, embedment and film perforation respectively.

\section{Weathering Experiments}

To assess pDCPD degradability under the marine environment, we exposed the material to a synthetic seawater matrix both in the dark and under simulated solar irradiation. We 
hypothesized that photooxidation of iPrSi-doped pDCPD would further enhance its aqueous wettability by introducing oxygen functional groups, assisting the hydrolysis of silyl ether groups. An approximately $100 \mathrm{mg}$ polymer pellet was submerged in the bottom of $60 \mathrm{~mL}$ of synthetic seawater matrix in a clear vial sealed with PTFE-lined cap. The synthetic seawater recipe was: $420 \mathrm{mM} \mathrm{NaCl}, 0.8 \mathrm{mM} \mathrm{NaBr}, 29 \mathrm{mM} \mathrm{Na}_{2} \mathrm{SO}_{4}, 54 \mathrm{mM} \mathrm{MgCl}{ }_{2} \cdot 6 \mathrm{H}_{2} \mathrm{O}, 11 \mathrm{mM}$ $\mathrm{CaCl}_{2} \cdot 2 \mathrm{H}_{2} \mathrm{O}, 10 \mathrm{mM} \mathrm{KCl}, 0.35 \mathrm{mM} \mathrm{H}_{3} \mathrm{BO}_{3}, 1.8 \mathrm{mM} \mathrm{NaHCO}_{3}$, and $0.26 \mathrm{mM} \mathrm{Na}_{2} \mathrm{CO}_{3}, 5$ $\mathrm{nM} \mathrm{FeCl}{ }_{3} 41$.

The vial was then exposed placed within a Rayonet photoreactor with Hg lamps and an output spectrum shown in Extended Data Fig. 6a-b (measured by OceanInsight FLAME-SXR1-ES spectroradiometer) for $16 \mathrm{~d}$. Note that glass containers were used to filter sub-300$\mathrm{nm}$ light to more closely emulate the solar distribution (represented by ASTM 177 reference spectra). The temperature was controlled in both the light and dark experiments to $45-46{ }^{\circ} \mathrm{C}$, where the temperature inside the Rayonet reactor was monitored and the dark experiments were kept in an oven maintained at $45-46{ }^{\circ} \mathrm{C}$.

We calculated degradation as the mass of carbon released in the seawater solution over the mass of carbon in the original material. Total organic carbon was measured as non-purgable organic carbon by a Shimazu TOC-5000. Prior to analysis, the sample was acidified with $50 \% \mathrm{HCl}$ to $\mathrm{pH}<3$ and sparged with $\mathrm{N}_{2}$ for 8 min to remove inorganic carbon in seawater matrix. Total organic carbon in the virgin polymer was inferred from the chemical formulas and mass fractions of $\mathbf{i P r S i}$ or $\mathbf{E t S i}$ co-monomers. A paired t-Test was performed to determine whether content of $\mathbf{i P r S i}$ or light exposure has an impact on degradability.

\section{Recycling of pDCPD Fragments}

pDCPD fragments derived from 10\% iPrSi-doped pDCPD were prepared following the standard workflow described above. $500 \mathrm{mg}$ of the fragments were then dissolved in $1.5 \mathrm{~g}$ of DCPD, forming a viscous brown liquid. To this liquid was added $8 \mathrm{mg}$ of finely powdered Grubbs' $2^{\text {nd }}$ generation ROMP initiator. The material was poured into vials (for forming discs) and silicone molds (for tensile and DMA measurements) and cured at $120{ }^{\circ} \mathrm{C}$ for 30 $\min$.

\section{Extended Data}


a

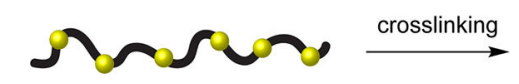

strand with cleavable main chain $f$ potential crosslinking sites

$x$ cleavable bonds

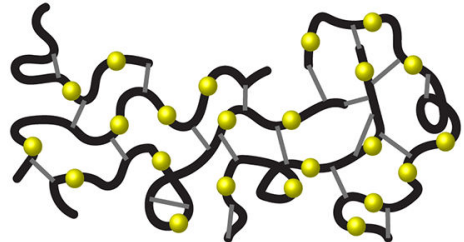

thermoset network c crosslinks per strand

b

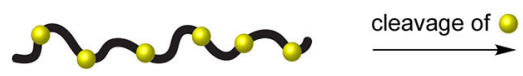

strand with cleavable main chain $f$ potential crosslinking sites

$x$ cleavable bonds

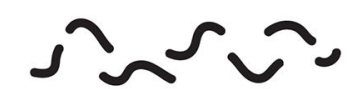

linear degradation products $<f /(x+1)>$ crosslinking sites per fragment
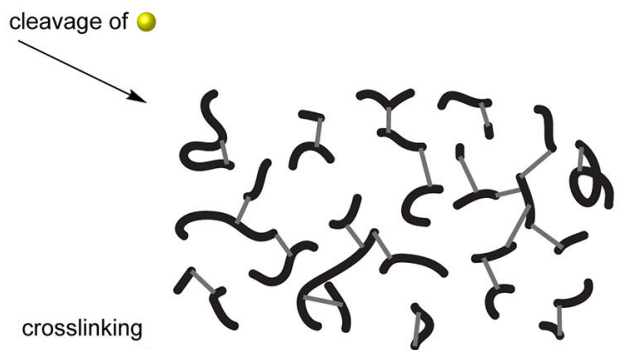

crosslinking

crosslinked degradation products $<c /(x+1)>$ crosslinks per fragment

Extended Data Figure 1. Schematic describing the reverse gel-point concept used to derive the model of degradable thermosets shown in Figure $\mathbf{1 b}$.

a. A thermoset network containing $f$ potential crosslinks per strand, $c$ actual crosslinks per strand, and $x$ cleavable bonds within each strand may or may not be degraded into soluble fragments after bond cleavage. A model determining whether the material will dissolve can be described as a function of $f, c$, and $x$ (Fig. 1b). b. The reverse gel-point concept enables this model by assuming that the minimum value $x$ required to enable thermoset degradation for a given $c$ and $f$ is the same value that will inhibit the gelation of degradation fragments derived from strands with $f$ potential crosslinking sites and $x$ cleavable bonds. We leverage existing gelation theories (Miller-Macosko and Flory-Stockmayer) to solve for $x$ given $f$ and c. Key to the reverse gel-point concept is the assumption that the network structure formed by the crosslinking of linear copolymer strands followed by cleavage of degradable bonds in those strands is identical to the network formed by first cleaving the linear copolymer strands and then cross-linking the resulting degradation products. 
a $\quad$ b

b
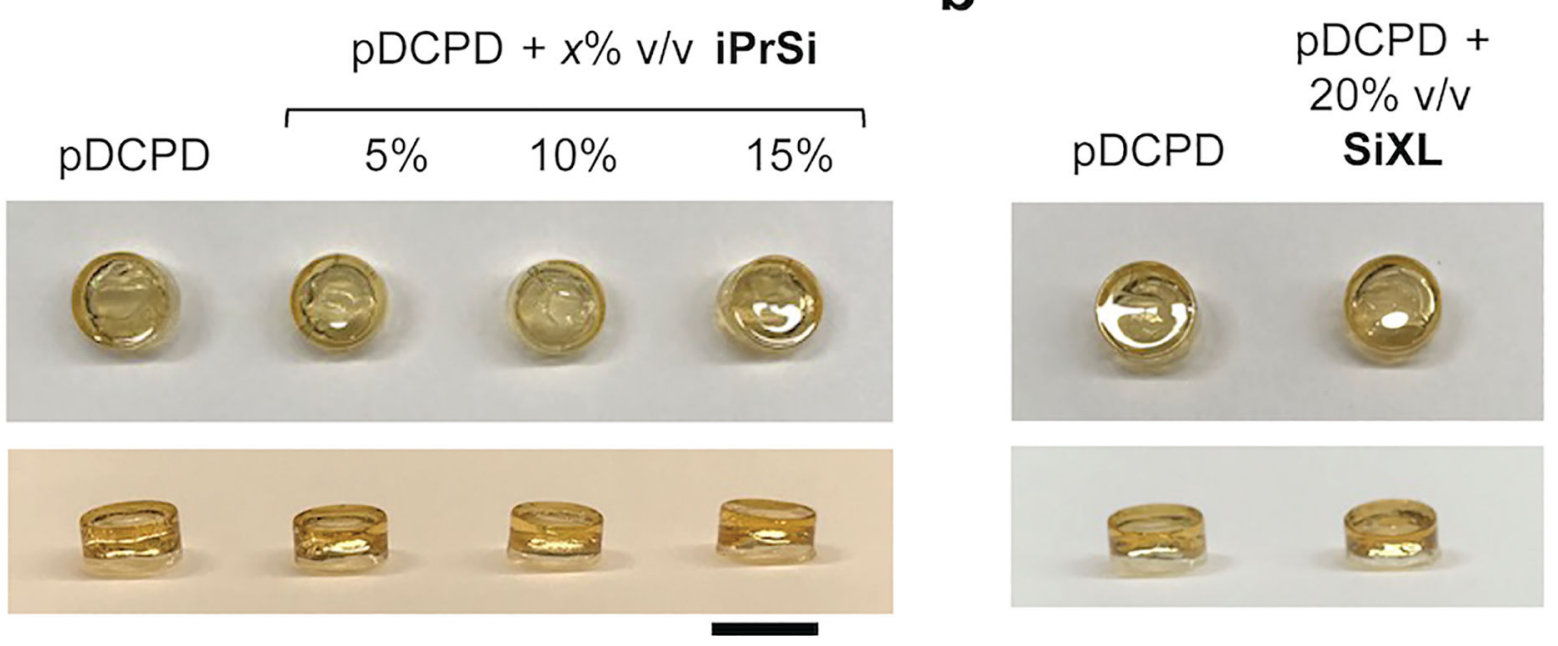

C
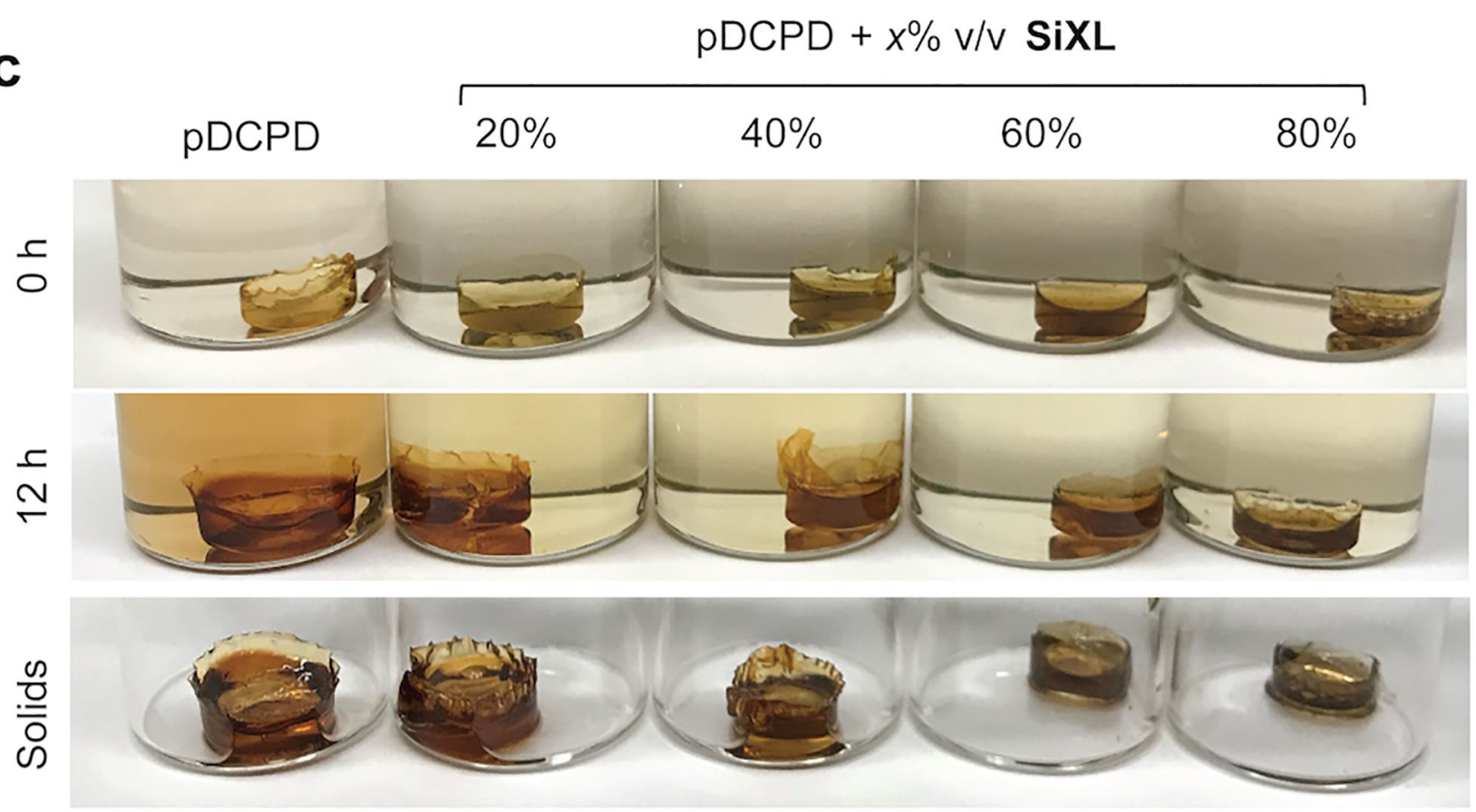

Extended Data Figure 2. Characterization of pDCPD

a. Images of $\mathrm{pDCPD}$ with or without various amounts of iPrSi. b. Images of pDCPD with or without $20 \% \mathrm{v} / \mathrm{v}$ of SiXL. c. pDCPD doped with up to $80 \% \mathrm{v} / \mathrm{v}$ SiXL remains intact after 12 h TBAF treatment. 
a

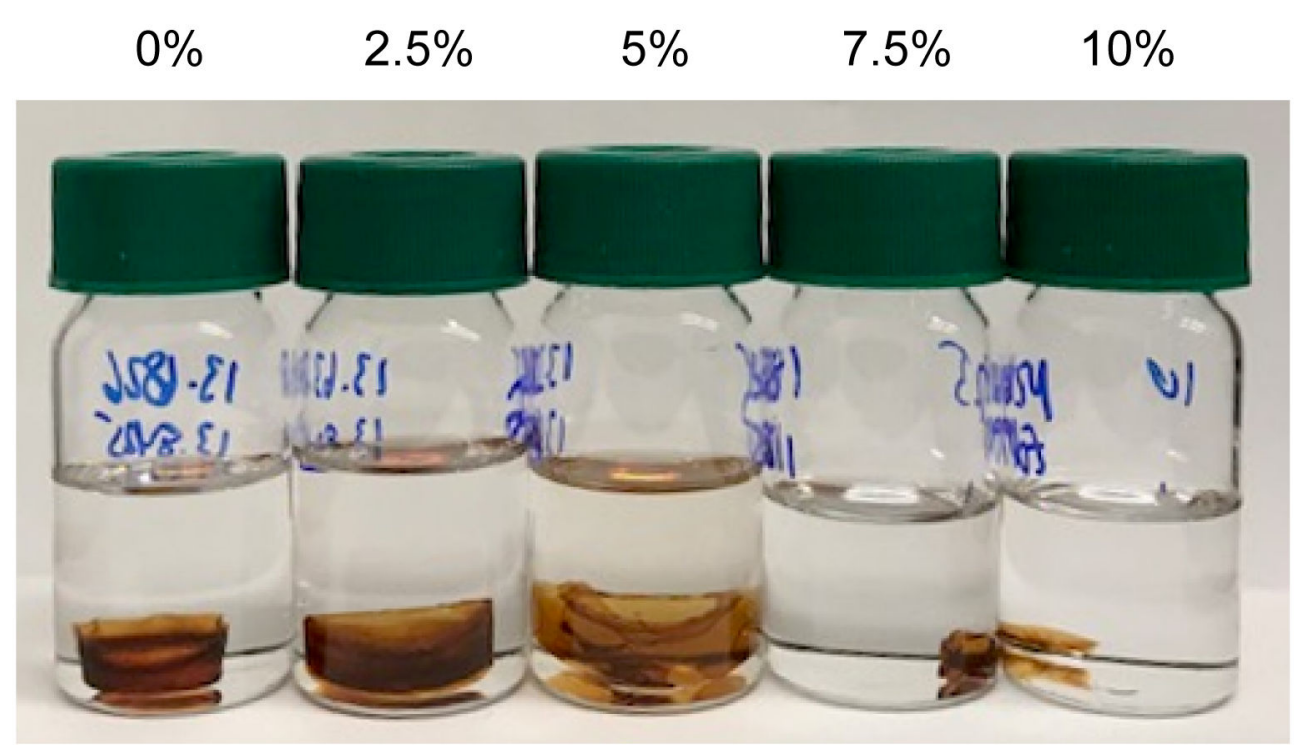

b
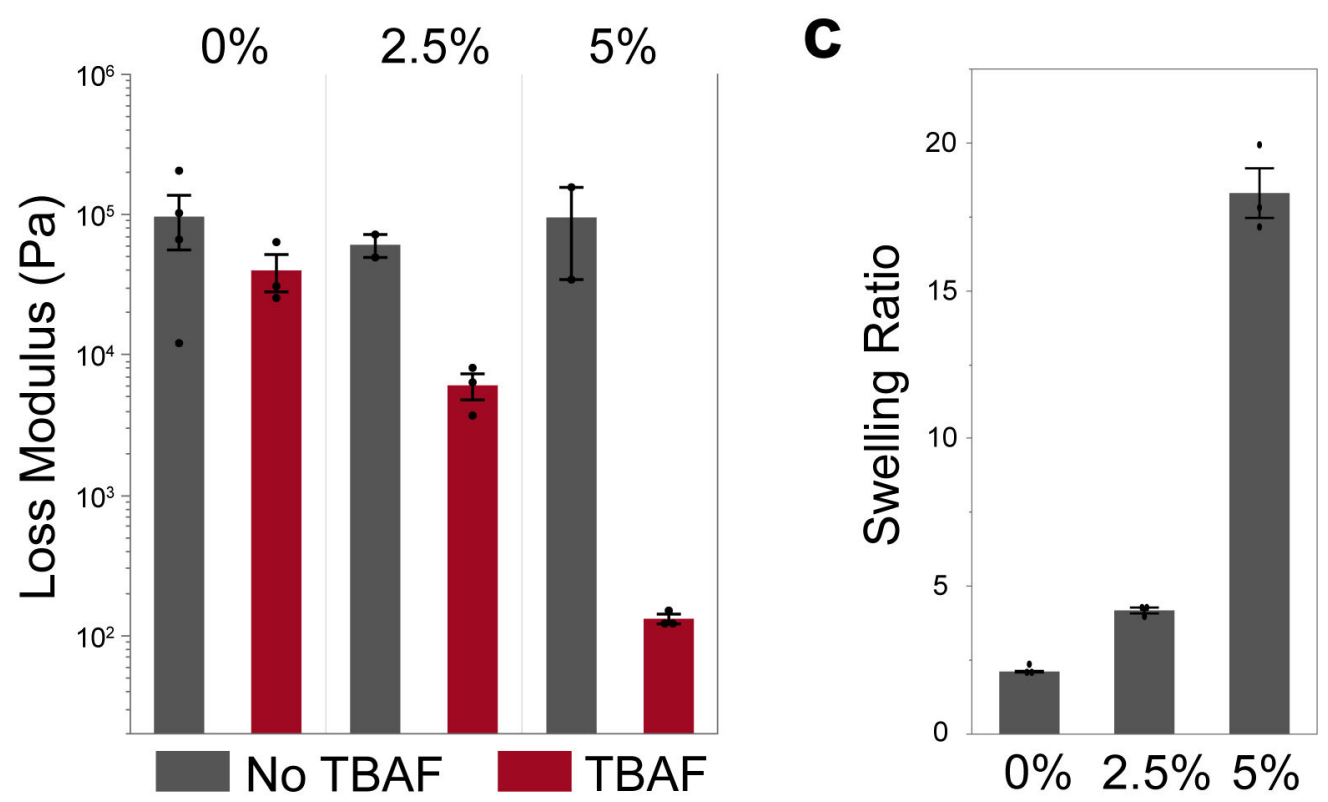

Extended Data Figure 3. Further quantification of the impact of silyl ether incorporation into pDCPD strands.

a. Samples containing different amounts of $\mathbf{i P r S i}(0,2.5,5,7.5$, and $10 \% \mathrm{v} / \mathrm{v})$ were incubated in 0.5 M TBAF in THF overnight, showing iPrSi-dependent degradation. b. Loss moduli for native pDCPD and $2.5 \%$ and $5 \%$ iPrSi-doped samples before and after TBAF treatment as measured by oscillatory rheology. Storage moduli are presented in Fig. 2c. c. THF swelling ratios (THF swollen mass divided by dry mass) for native pDCPD and $2.5 \%$ and $5 \%$ iPrSi-doped samples following TBAF treatment. $n=3$ for all samples. 
a

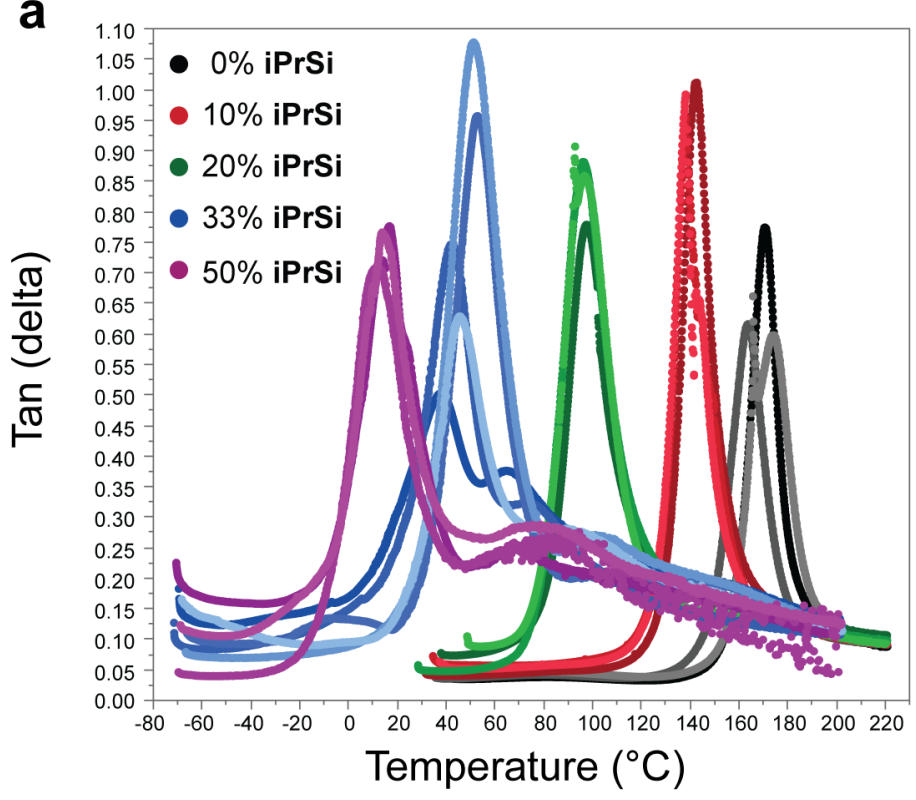

b

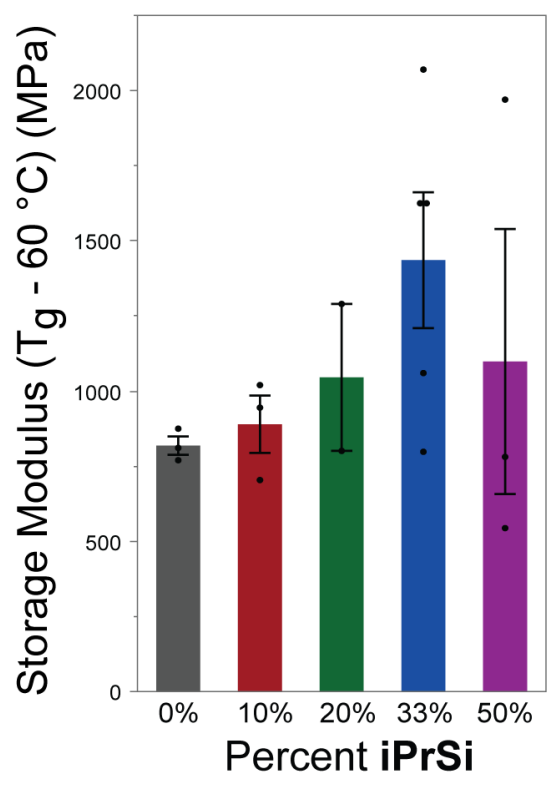

Extended Data Figure 4. Characterization of mechanical and thermal properties of iPrSi-doped pDCPD by DMA.

a. Tan-delta plots of pDCPD samples as a function of iPrSi incorporation. b. Storage moduli collected at $\mathrm{T}_{\mathrm{g}}-60^{\circ} \mathrm{C}$ for all samples. $n=3$ for all samples, except for $33 \%$ where $n=5$. 
a

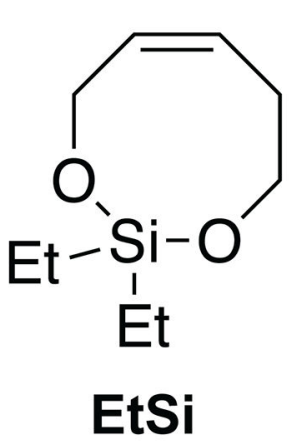

b

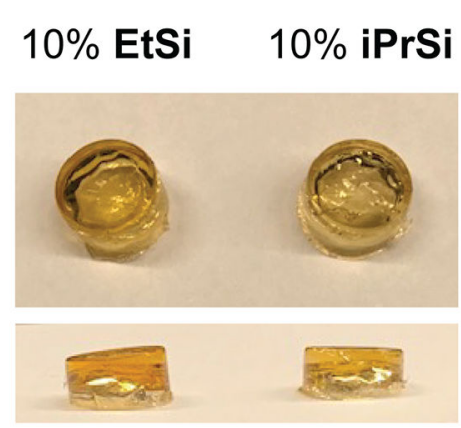

C

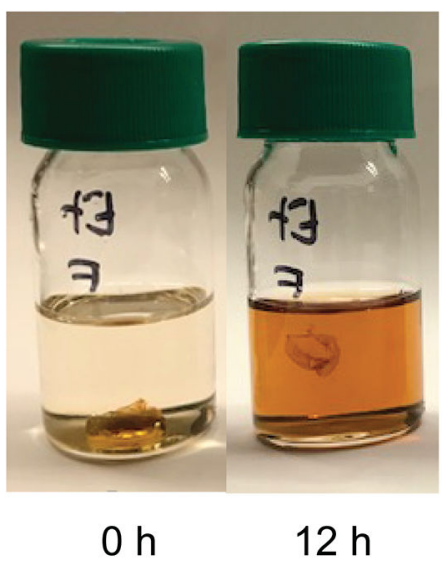

d

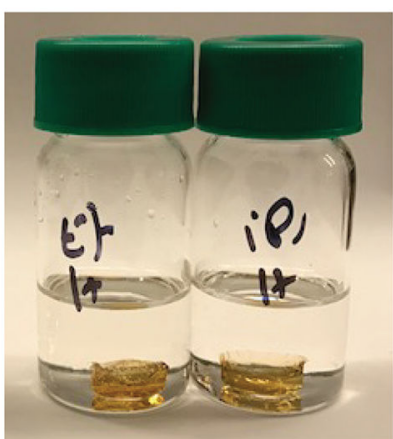

$\mathrm{Oh}$

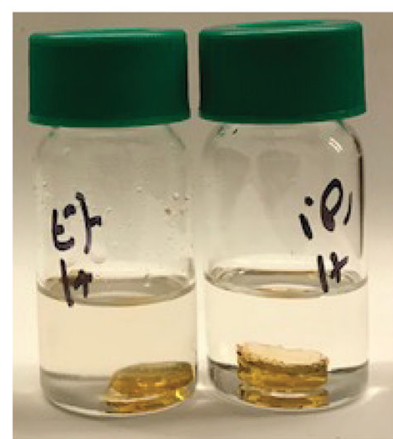

$15 \min$

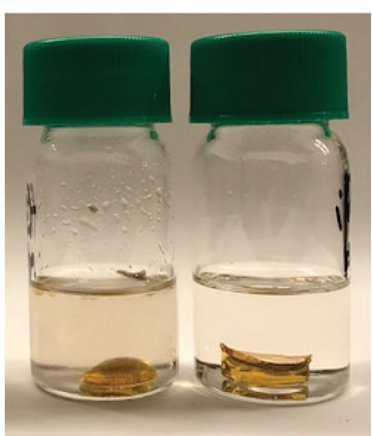

$1 \mathrm{~h}$

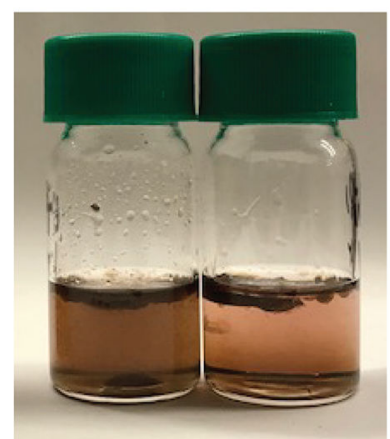

$12 \mathrm{~h}$

Extended Data Figure 5. Synthesis and degradation of EtSi or iPrSi doped pDCPD.

a. Structure of EtSi, which differs from iPrSi in terms of the alkyl substituents on the silyl ether group. The less sterically hindered ethyl groups render this monomer more susceptible to cleavage. b. Images of $10 \%$ EtSi or iPrSi doped pDCPD. c. $10 \%$ EtSi dissolves in $0.5 \mathrm{M}$ TBAF in THF after 12 h. d. Images of $10 \%$ EtSi (left) or iPrSi (right)-doped pDCPD exposed to THF containing $15 \%$ concentrated aqueous $\mathrm{HCl}(12.1 \mathrm{~N})$. The EtSi sample shows noticeably more rapid degradation under these conditions as compared to the iPrSi sample. Both samples are largely degraded by $12 \mathrm{~h}$. In this case, acidic hydrolysis is facilitated by the presence of organic solvent to swell the network. 

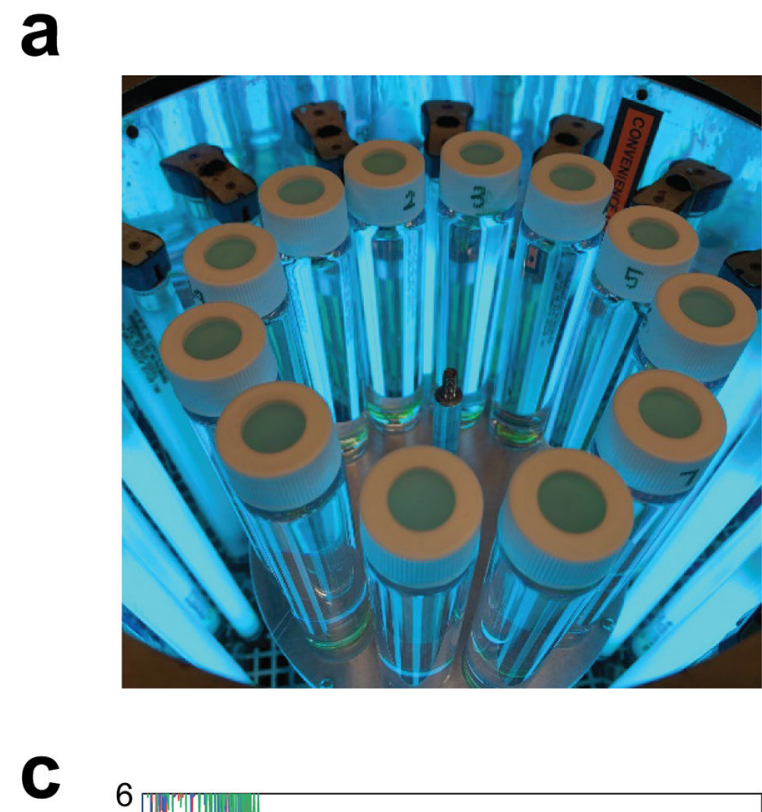

Extended Data Figure 6. Weathering studies

\section{Supplementary Material}

\section{Acknowledgements}

b

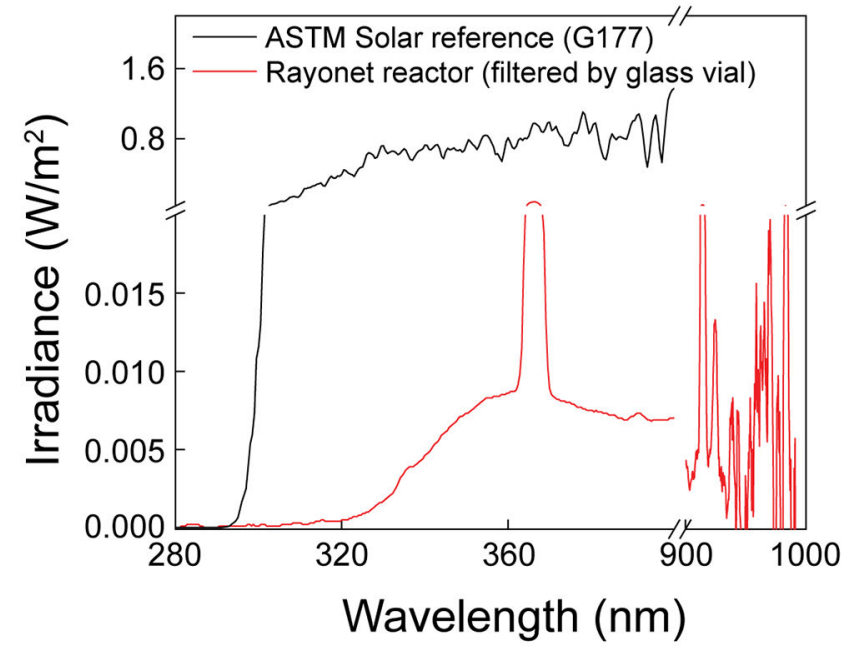

d
Light

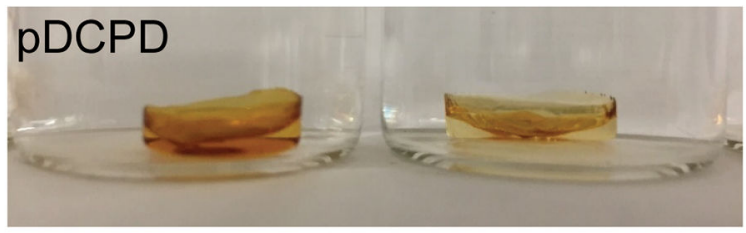

$+10 \%$ iPrSi

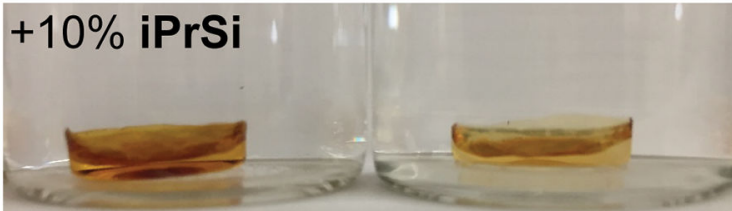

a. Image of weathering setup. Samples were kept inside glass vials over the course of weathering experiments. b. Measured irradiance for samples during weathering experiments and comparison to solar reference spectra (ASTM G177). c. UV-vis spectra for the 0\%, $10 \%$, and $20 \%$ iPrSi and 10\% EtSi doped pDCPD samples. Sample thickness was $1 \mathrm{~mm}$. d. Images of samples before and after weathering studies.

Refer to Web version on PubMed Central for supplementary material.

The authors thank the National Science Foundation (DMREF CHE-1629358) and the National Institutes of Health (1R01CA220468-01) for support of this work. P.S. was supported by a fellowship from the American Cancer Society. S.L.K. was supported by a fellowship from the Misrock Foundation. We thank Dr. Bruce Adams and Dr. Walt Massefski for assistance with NMR analysis, Dr. Alan Schwartzman for assistance with AFM and nanoindentation measurements, Dr. Timothy McClure for assistance with ICP-OES measurements, Michael 
Tarkanian for assistance with mold fabrication, and Shao-Xiong Luo for assistance with Raman measurements. J.L., D.V., Y.S. and K.A.N. acknowledge support for the microparticle impact experiments from the U. S. Army Research Office through the Institute for Soldier Nanotechnologies, under Cooperative Agreement Numbers W911NF-18-2-0048.

Competing interests

P.S., W.Z., K.E.L.H., and J.A.J. are named inventors on patent applications filed by the Massachusetts Institute of Technology on the copolymers described in this work.

\section{References}

1. Ma S \& Webster DC Degradable thermosets based on labile bonds or linkages: A review. Prog. Polym. Sci 76, 65-110 (2018).

2. Post W, Susa A, Blaauw R, Molenveld K \& Knoop RJI A Review on the Potential and Limitations of Recyclable Thermosets for Structural Applications. Polym. Rev 1-30 (2019). doi:10.1080/15583724.2019.1673406

3. Kloxin CJ, Scott TF, Adzima BJ \& Bowman CN Covalent adaptable networks (CANs): A unique paradigm in cross-linked polymers. Macromolecules 43, 2643-2653 (2010). [PubMed: 20305795]

4. Gu Y, Zhao J \& Johnson JA Polymer Networks: From Plastics and Gels to Porous Frameworks. Angew. Chemie - Int. Ed 59, 5022-5049 (2020).

5. Winne JM, Leibler L \& Du Prez FE Dynamic covalent chemistry in polymer networks: A mechanistic perspective. Polym. Chem 10, 6091-6108 (2019).

6. Montarnal D, Capelot M, Tournilhac F \& Leibler L Silica-like malleable materials from permanent organic networks. Science 334, 965-968 (2011). [PubMed: 22096195]

7. Röttger $\mathrm{M}$ et al. High-performance vitrimers from commodity thermoplastics through dioxaborolane metathesis. Science 356, 62-65 (2017). [PubMed: 28386008]

8. Li L, Chen X, Jin K \& Torkelson JM Vitrimers Designed Both to Strongly Suppress Creep and to Recover Original Cross-Link Density after Reprocessing: Quantitative Theory and Experiments. Macromolecules 51, 5537-5546 (2018).

9. Asaro L, Gratton M, Seghar S \& Aït Hocine N Recycling of rubber wastes by devulcanization. Resour. Conserv. Recycl 133, 250-262 (2018).

10. Yang S et al. Reworkable Epoxies: Thermosets with Thermally Cleavable Groups for Controlled Network Breakdown. Chem. Mater 10, 1475-1482 (1998).

11. Christensen PR, Scheuermann AM, Loeffler KE \& Helms BA Closed-loop recycling of plastics enabled by dynamic covalent diketoenamine bonds. Nat. Chem 11, 442-448 (2019). [PubMed: 31011169]

12. Li L, Chen X \& Torkelson JM Reprocessable Polymer Networks via Thiourethane Dynamic Chemistry: Recovery of Cross-link Density after Recycling and Proof-of-Principle Solvolysis Leading to Monomer Recovery. Macromolecules 52, 8207-8216 (2019).

13. Fortman DJ, Brutman JP, Cramer CJ, Hillmyer MA \& Dichtel WR Mechanically Activated, Catalyst-Free Polyhydroxyurethane Vitrimers. J. Am. Chem. Soc 137, 14019-14022 (2015). [PubMed: 26495769]

14. Rule JD \& Moore JS ROMP reactivity of endo- and exo-dicyclopentadiene. Macromolecules 35, 7878-7882 (2002).

15. Kessler MR \& White SR Cure kinetics of the ring-opening metathesis polymerization of dicyclopentadiene. J. Polym. Sci. Part A Polym. Chem 40, 2373-2383 (2002).

16. Robertson ID et al. Rapid energy-efficient manufacturing of polymers and composites via frontal polymerization. Nature 557, 223-227 (2018). [PubMed: 29743687]

17. Shieh P, Nguyen HVT \& Johnson JA Tailored silyl ether monomers enable backbone-degradable polynorbornene-based linear, bottlebrush and star copolymers through ROMP. Nat. Chem 11, 1124-1132 (2019). [PubMed: 31659310]

18. Wang B, Ma S, Yan S \& Zhu J Readily recyclable carbon fiber reinforced composites based on degradable thermosets: A review. Green Chem. 21, 5781-5796 (2019). 
19. Fortman DJ et al. Approaches to Sustainable and Continually Recyclable Cross-Linked Polymers. ACS Sustain. Chem. Eng 6, 11145-11159 (2018).

20. Takahashi A, Ohishi T, Goseki R \& Otsuka H Degradable epoxy resins prepared from diepoxide monomer with dynamic covalent disulfide linkage. Polymer 82, 319-326 (2016).

21. Wiles DM \& Scott G Polyolefins with controlled environmental degradability. Polym. Degrad. Stab 91, 1581-1592 (2006).

22. Sommazzi A \& Garbassi F Olefin-carbon monoxide copolymers. Progress in Polymer Science (Oxford) 22, 1547-1605 (1997).

23. Macosko CW \& Miller DR A New Derivation of Average Molecular Weights of Nonlinear Polymers. Macromolecules 9, 199-206 (1976). [PubMed: 1263575]

24. Flory PJ Molecular Size Distribution in Three Dimensional Polymers. I. Gelation. J. Am. Chem. Soc 63, 3083-3090 (1941).

25. Stockmayer WH Theory of molecular size distribution and gel formation in branched polymers: II. General cross linking. J. Chem. Phys 12, 125-131 (1944).

26. Wang $\mathrm{J}$ et al. Counting loops in sidechain-crosslinked polymers from elastic solids to single-chain nanoparticles. Chem. Sci 10, 5332-5337 (2019). [PubMed: 31191890]

27. Takayama $\mathrm{S}$ et al. Topographical micropatterning of poly(dimethylsiloxane) using laminar flows of liquids in capillaries. Adv. Mater 13, 570-574 (2001).

28. Long TR et al. Ballistic response of polydicyclopentadiene vs. epoxy resins and effects of crosslinking in Conference Proceedings of the Society for Experimental Mechanics Series 1B, 285-290 (Springer New York LLC, 2017).

29. Veysset D et al. Dynamics of supersonic microparticle impact on elastomers revealed by real-time multi-frame imaging. Sci. Rep 6, (2016).

30. Davies JS, Higginbotham CL, Tremeer EJ, Brown C \& Treadgold RC Protection of hydroxy groups by silylation: use in peptide synthesis and as lipophilicity modifiers for peptides. J. Chem. Soc. Perkin Trans. 13043 (1992). doi:10.1039/p19920003043

31. Tournier $\mathrm{V}$ et al. An engineered PET depolymerase to break down and recycle plastic bottles. Nature 580, 216-219 (2020). [PubMed: 32269349]

32. Cole M, Lindeque P, Halsband C \& Galloway TS Microplastics as contaminants in the marine environment: A review. Marine Pollution Bulletin 62, 2588-2597 (2011). [PubMed: 22001295]

33. Hann S, Ettlinger S, Gibbs A \& Hogg D The Impact of the Use of 'Oxo-degradable' Plastic on the Environment. European Commission (2016). doi:10.2779/992559

34. Autenrieth B et al. Stereospecific ring-opening metathesis polymerization (ROMP) of endodicyclopentadiene by molybdenum and tungsten catalysts. Macromolecules 48, 2480-2492 (2015).

35. Chen J, Burns FP, Moffitt MG \& Wulff JE Thermally Crosslinked Functionalized Polydicyclopentadiene with a High Tg and Tunable Surface Energy. ACS Omega 1, 532-540 (2016). [PubMed: 31457145]

36. Flory PJ Random Reorganization of Molecular Weight Distribution in Linear Condensation Polymers. J. Am. Chem. Soc 64, 2205-2212 (1942).

37. Lee JH et al. High strain rate deformation of layered nanocomposites. Nat. Commun 3, (2012).

38. Imbriglio SI et al. Adhesion strength of titanium particles to alumina substrates: A combined cold spray and LIPIT study. Surf. Coatings Technol 361, 403-412 (2019).

39. Sun $Y$ et al. Molecular dependencies of dynamic stiffening and strengthening through high strain rate microparticle impact of polyurethane and polyurea elastomers. Appl. Phys. Lett 115, (2019).

40. Veysset D, Hsieh AJ, Kooi SE \& Nelson KA Molecular influence in high-strain-rate microparticle impact response of poly(urethane urea) elastomers. Polymer 123, 30-38 (2017).

41. Parker KM \& Mitch WA Halogen radicals contribute to photooxidation in coastal and estuarine waters. Proc. Natl. Acad. Sci. U. S. A 113, 5868-5873 (2016). [PubMed: 27162335] 
a

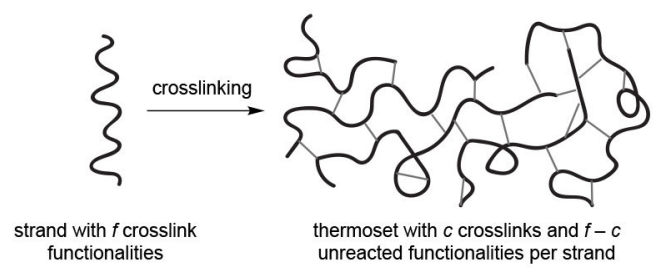

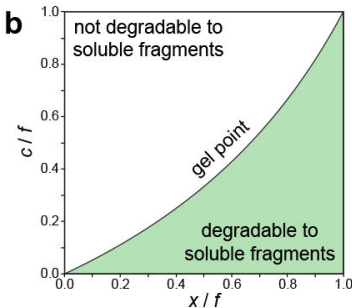

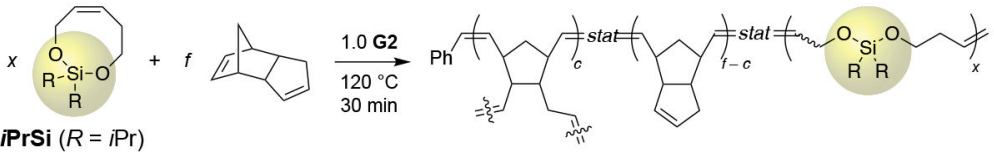
EtSi $(R=\mathrm{Et})$
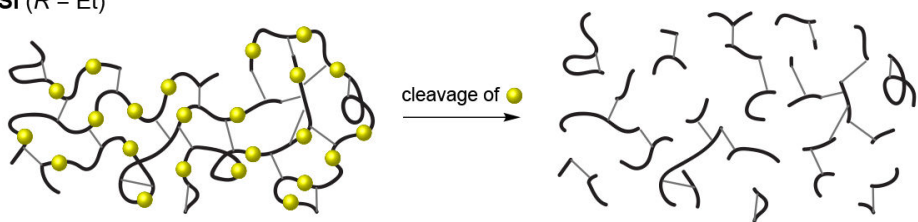

degradation fragments c crosslinks per strand $x$ cleavable bonds

$c /(x+1)$ crosslinks per fragment
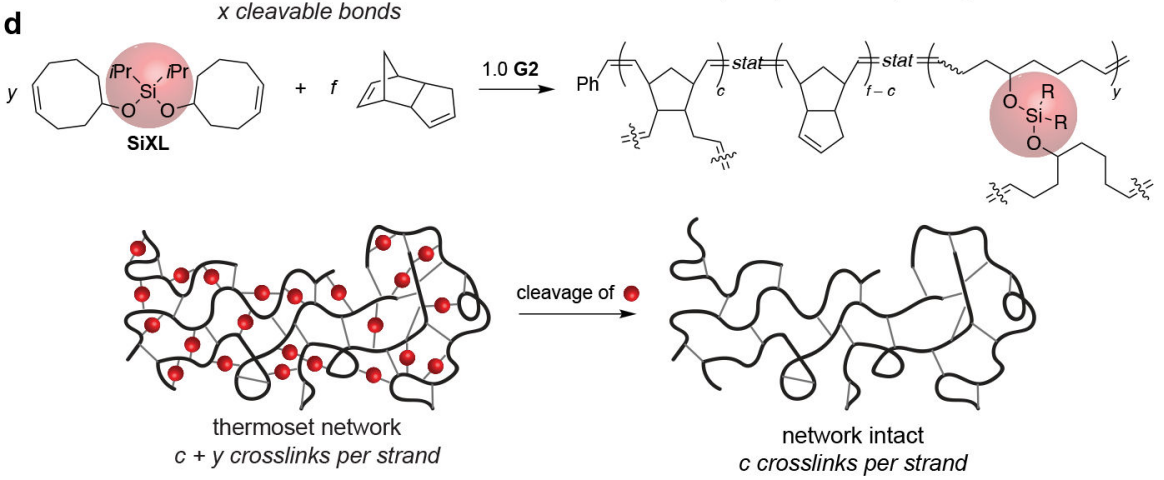

Figure 1. Conceptual basis of this work.

a. Thermosets are often synthesized from the crosslinking of linear prepolymers with $f$ crosslink functionalities. The resulting materials can have outstanding properties, but they often have unknown numbers of crosslinks $(c)$ and they are typically non-degradable/nonreprocessable. $\mathbf{b}$. Theoretical model describing what amounts of cleavable monomer $(x)$ relative to non-cleavable crosslinks $(c)$ will result in degradation of materials composed of strands of functionality $f=3000$ into soluble products. c. The silyl ether-based monomer iPrSi co-polymerizes efficiently with norbornenes through ROMP, which introduces cleavage sites within the polynorbornene strands of pDCPD. The introduction of $x$ cleavable bonds within the strands of pDCPD with $c$ crosslinks provides degradation fragments with $<c /(x+1)>$ crosslinks per strand. d. The silyl ether crosslinker SiXL co-polymerizes with norbornenes through ROMP, but instead introduces cleavage sites between polynorbornene strands (i.e., in crosslinks). The introduction of $y$ cleavable crosslinks produces pDCPD with $c+y$ crosslinks. Thus, soluble products can only be generated when $y>c$, suggesting that complete material degradation at low co-monomer loading is difficult. 
a

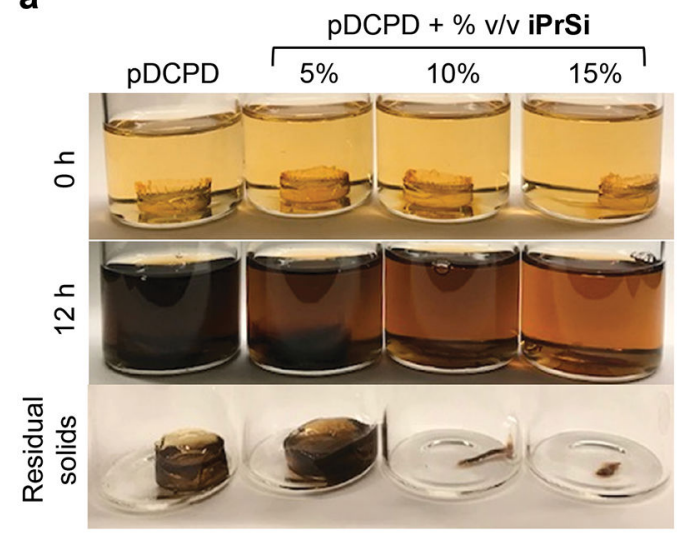

b

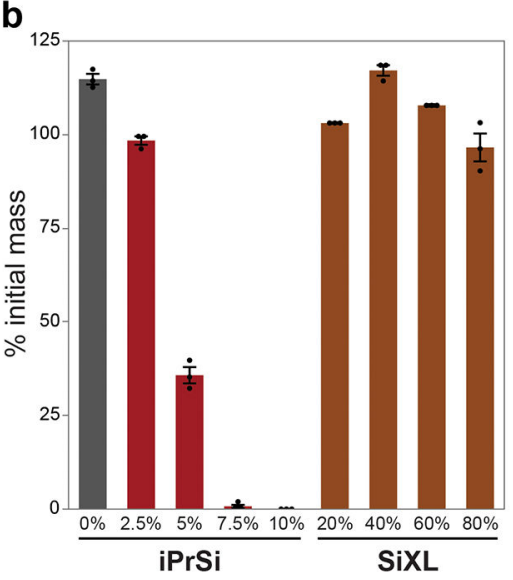

C

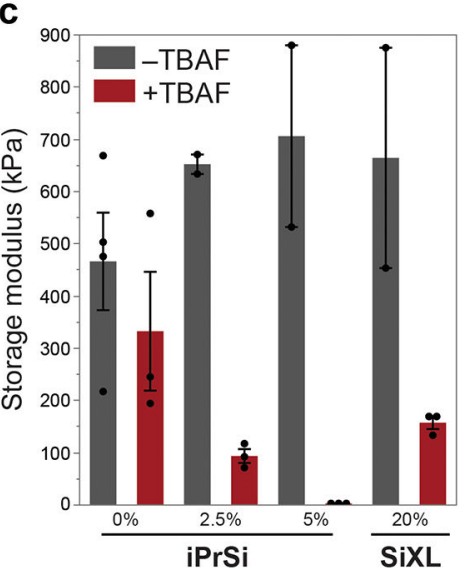

Figure 2. Precise placement of a small number of degradable bonds within the strands of thermoset plastic enables degradation into soluble products.

a. iPrSi-Doped pDCPD samples show iPrSi volume-fraction-dependent dissolution in a THF solution of tetrabutylammonium fluoride (TBAF). Samples containing 10 or $15 \% \mathbf{i P r S i}$ are almost entirely dissolved. Treatment with HF provides similar results. b. Quantification of residual mass of pDCPD samples after TBAF treatment at room temperature for $12 \mathrm{~h}$ or $17 \mathrm{~d}$ for $\mathbf{i P r S i}$ - or SiXL-doped samples, respectively. The 7.5\% and $10 \%$ iPrSi-doped samples show nearly complete mass loss while samples prepared with up to $80 \%$ of SiXL remained intact. c. Shear rheology on THF-swollen samples shows a more dramatic TBAFdependent loss in storage modulus for samples containing small amounts $(2.5 \%$ and $5 \% \mathrm{v} / \mathrm{v})$ of iPrSi compared to $20 \% \mathrm{v} / \mathrm{v}$ of SiXL, highlighting the greater contribution of cleavable strands to network integrity. Samples were exposed to TBAF for $12 \mathrm{~h}$ at room temperature. Error bars - mean + /- S.E.M, $n=3$ for mass quantification, $n=2-4$ for rheology. 

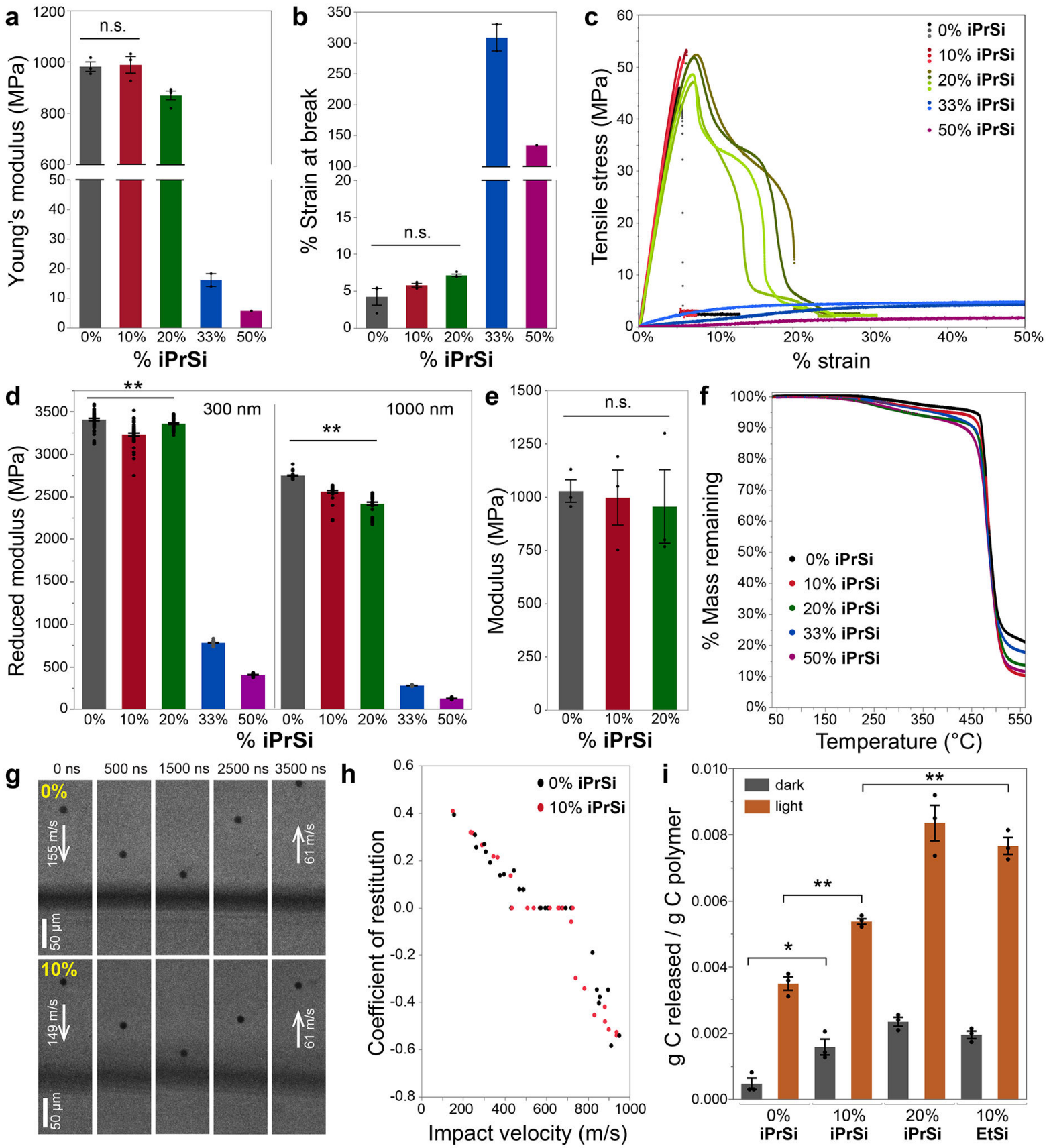

Figure 3. Functional evaluation of doped pDCPD.

a. iPrSi-doped pDCPD shows no significant difference in Young's modulus (measured at room temperature) between 0 and $10 \%$ iPrSi. The $33 \%$ and $50 \%$ iPrSi-doped samples are closer to or above their $\mathrm{T}_{\mathrm{g}}$ at room temperature $\left(46^{\circ} \mathrm{C}\right.$ and $14{ }^{\circ} \mathrm{C}$, respectively), which explains their different tensile behaviors compared to native pDCPD. b. iPrSi-doped pDCPD shows no significant difference in strain at break at low iPrSi loadings. c. Stressstrain curves obtained at room temperature for iPrSi-doped pDCPD samples and native pDCPD highlighting the similarity between 10\% iPrSi-doped sample and native pDCPD as well as the ability to control stress-strain behavior with comonomer loading. d. iPrSi-doped 
pDCPD samples show comparable reduced moduli to native pDCPD as assessed by nanoindentation. e. iPrSi-doped pDCPD shows no statistically significant difference in modulus compared to pDCPD at low iPrSi loadings by dynamic mechanical analysis. f. iPrSi-doped pDCPD shows similar decomposition temperatures as native pDCPD. $\mathbf{g}$. Representative image sequences of impact and rebound for $0 \%$ and $10 \%$ iPrSi-doped pDCPD impacted by steel microparticles. h. Coefficient of restitution (CoR) plots for $0 \%$ and $10 \%$ iPrSi-doped pDCPD. Positive, zero, and negative CoR correspond to particle rebound, embedment, and film perforation, respectively. i. iPrSi and EtSi doping allows for tuning of degradation in synthetic seawater as assessed by analysis of dissolved carbon. These results suggest that the comonomer approach could allow for optimization of degradation for applications of interest. n.s. $-\mathrm{P}>0.1, *-\mathrm{P}<0.05, * *-\mathrm{P}<0.01$. Error bars - mean + - S.E.M. $n=3$ for $0 \%$ and $10 \%, n=4$ for $20 \%, n=2$ for $33 \%$, and $n=1$ for $50 \%$ for tensile testing, $n=46-49$ for nanoindentation, $n=3$ for DMA, and $n=3$ for weathering experiments. 


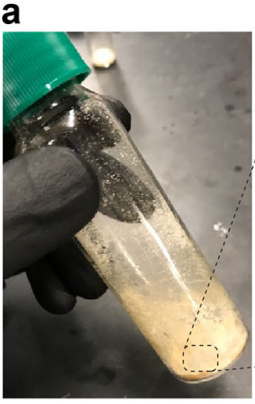

C
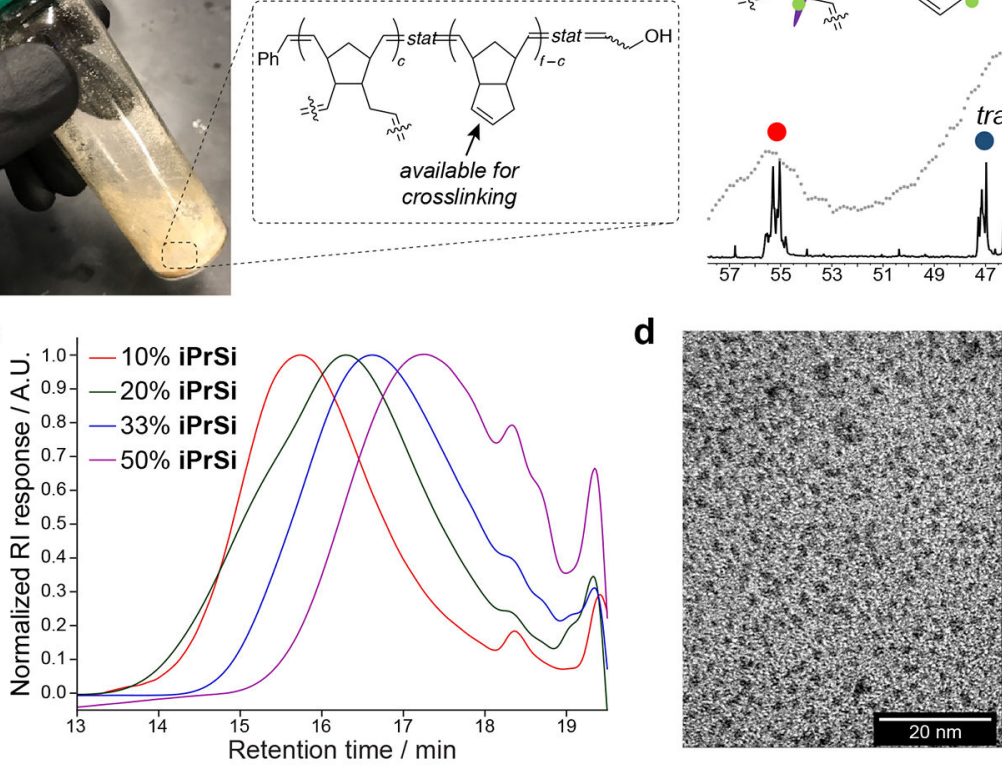

b
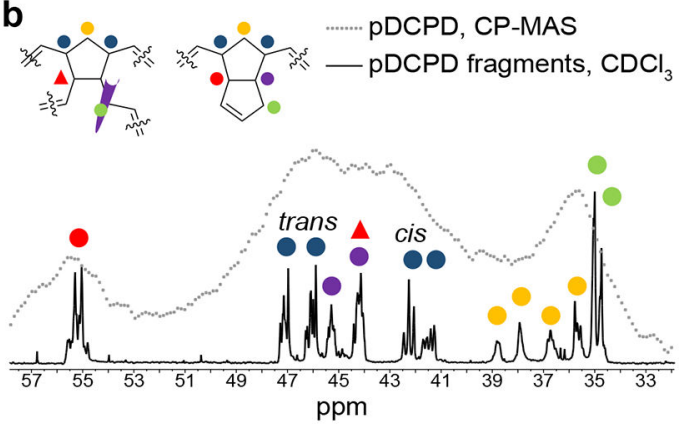

$\mathrm{ppm}$

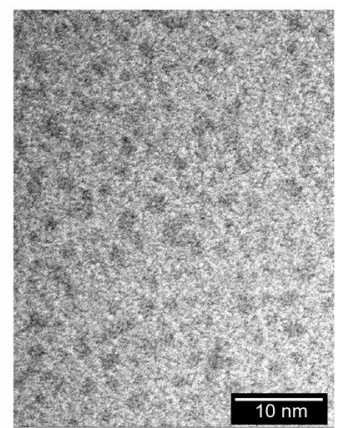

e
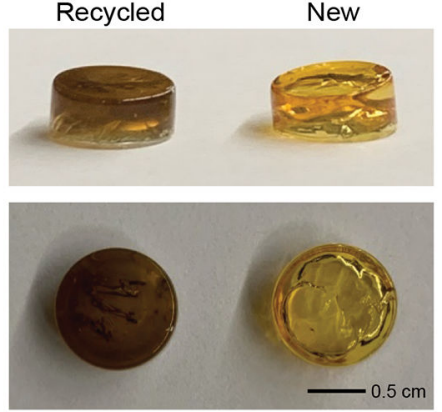

h

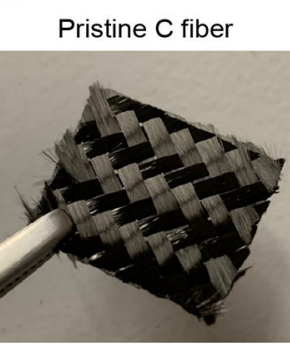

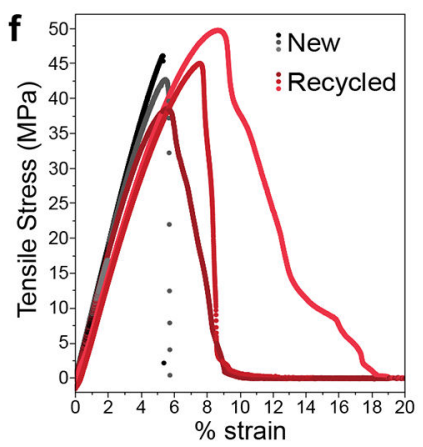

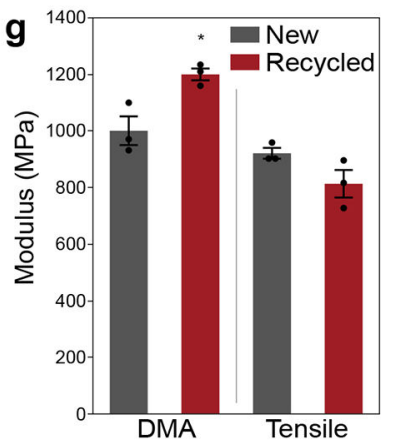

i

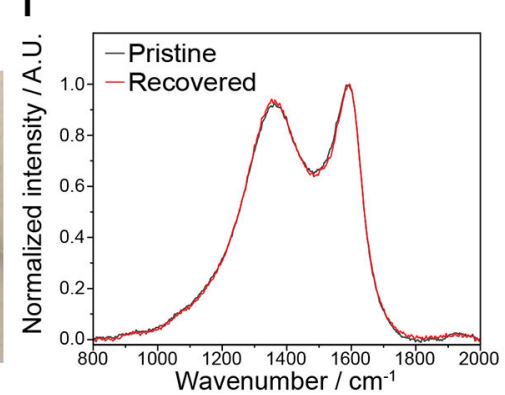

Figure 4. Soluble pDCPD fragments enable high-resolution characterization of pDCPD and can be recycled into new materials.

a. Image of dried pDCPD fragments from $10 \%$ iPrSi-doped material. These soluble, processable fragments possess a large number of cyclopentene functional groups available for further crosslinking. b. Comparison of ${ }^{13} \mathrm{C} N M R$ spectra of $\mathrm{pDCPD}$ derived from CPMAS (traditional approach for thermoset characterization) or from analysis of soluble degradation products from $10 \%$ iPrSi-doped sample. The soluble products display improved spectral resolution, enabling assignment and characterization of the structure of iPrSi-doped pDCPD. c. GPC traces of fragments derived from the dissolution of iPrSi-doped pDCPD. 
As expected, increased iPrSi loading leads to smaller degradation products as evidenced by increases in retention time. d. TEM images of fragments derived from $10 \% \mathrm{v} / \mathrm{v}$ iPrSi-doped pDCPD showing an average particle size of $\sim 4 \mathrm{~nm}$. e. Images of representative recycled and new pDCPD samples. Discoloration of the recycled sample is attributed to residual Ru from the first crosslinking and degradation process. f. Stress-strain curves from dogbone-shaped samples of new and recycled pDCPD, showing comparable stress strain curves with increased strain at break for the recycled samples. g. Elastic moduli of native and recycled pDCPD as assessed by DMA and tensile testing. h. Images of carbon fiber recovery from $10 \%$ iPrSi-doped pDCPD composites. Costly carbon fiber filler is often unrecoverable from thermoset composites. The degradable comonomer approach allows for its recovery. $\mathbf{i}$. Raman spectra of pristine and recovered carbon fibers, suggesting no chemical damage to the carbon fiber material. Error bars - mean + /- S.E.M. $n=3$ for all conditions. 